\title{
LOW-INTENSITY MAGNETIC SEPARATION: PRINCIPAL STAGES OF A SEPARATOR DEVELOPMENT - WHAT IS THE NEXT STEP?
}

\author{
M.A. BIKBOV ${ }^{\mathrm{a}, *}$, V.V. KARMAZIN ${ }^{\mathrm{b}}$ and A.A. BIKBOV \\ ${ }^{a}$ Breakthrough Technologies Co. Ltd, Kostomuksha Mine Combine, Kostomuksha, Karelia, Russia; \\ ${ }^{\mathrm{b}}$ Moscow State Mining University, Moscow, Russia; ${ }^{\mathrm{c}}$ Ekaterinburg, Russia
}

(Received 24 March 2004; In final form 26 April 2004)

\begin{abstract}
An analysis of the technological limitations of magnetite quartzite beneficiation illustrated the imperfections of the traditional classification by size. As an alternative to size classification, separation by the degree of magnetite grain liberation can be employed. Comparative analysis of the mineral phase properties of the magnetic separation feed and its magnetic product has confirmed that wet drum magnetic separators currently used for wet treatment of magnetite ores have a low selectivity of separation. The removal of inter-grown particles into a separate product to enable more efficient and selective disintegration is expected to offer significant energy saving. A new-generation selective separator has been developed and a pilot model built; test results are promising. Grain selection by different degrees of mineral liberation is possible when the wet method is utilized. Magnetic selection allows for substantial benefits to be made through process optimization, including: (1) energy savings in the grinding process; (2) reduction of losses arising from over-ground magnetite in the operational tailings; (3) increased grain size; (4) avoidance of over-grinding; and (5) filtration and pelletization improvements.
\end{abstract}

Keywords: Magnetite; Quartzite; Beneficiation; Magnetic separation; Hydrocyclone; Liberation; Flocculation

\section{INTRODUCTION}

It is impossible not to take into consideration the current tendencies in the beneficiation of magnetite ores. During the last half-century, many of the conditions in which the process is carried out have changed. For instance, the state of the raw material base has deteriorated, as a result of the reduced ore grades, energy costs have risen, and environmental issues have become important. Additionally, metallurgical requirements for iron concentrate conditions have increased, as far as chemical composition and final concentrate stability are concerned [1].

The number and complexity of the above problems make significant demands on the industry and in order to meet these a thorough understanding of the tasks should be coupled with development of new technology. Such a promising approach will allow

*Corresponding author. E-mail: newgen@onego.ru 
us to utilize reserves that are, presently, exploited by imperfect technology of magnetite quartzite, taconite beneficiation.

It is the functional performance of the processing equipment that determines the possibility to realize certain technology. As a result of the existing equipment not being efficient, having many drawbacks and not being able to solve numerous problems, it is possible to claim that many existing technological barriers need to be broken [2].

\section{PRINCIPAL STAGES OF DEVELOPMENT OF A SEPARATOR}

The first attempts to apply permanent magnets to the dressing of magnetite ores date back to the 17 th century, as summarized by Derkach [3]. For instance, Rosler announced in 1700 the application of magnetic separation to treat cassiterite contaminated with iron, which could not be separated by water washing. The treatment was carried out by a hand magnet. In 1792 Fuller applied for a patent for the separation of iron ore using a magnet. In 1854 Palmer proposed a magnetic separator with magnet polarities alternating along the direction of the material movement.

Up until the end of the 19th century, methods of magnetic beneficiation were developed rather slowly, as a result of the absence of successful magnetic separator designs. The utilization of electromagnets to generate magnetic field for magnetic separation was suggested for the first time by Nonteponi in 1855 and served as a powerful incentive for further development.

A continuous separator, with a belt surrounding an electromagnetic drum and discharging magnetic material out of the action zone of the magnetic field into a concentrate bin, was first suggested in 1870. In 1884 a stationary magnetic system incompletely covering an internal drum surface was applied. Magnetic material was losing contact with the drum surface at this zone of reduced magnetic field.

Ball and Norton invented a separator with using magnetic agitation of material to be separated. The use of alternating polarities in the direction of magnetic particle movement became a significant achievement. In this case, magnetic particles and flocs rotate and release trapped non-magnetic particles, which results in the selective separation of magnetics from non-magnetics.

At the beginning of the 20th century, significant development took place in Sweden with the first wet magnetic separator being designed by Gröndal in 1906. Development of this separator, which served as a prototype for modern drum separators, allowed wet processing of fine sizes of magnetite ores successfully and profitably.

In 1922 Stearns suggested a separator using fine particle agitation in a magnetic field by means of periodical alternation of the direction of the electric current in the magnetization coils. A separator, in which a material is subjected to an action of alternating magnetic field and centrifugal force to improve magnetic separation of ground ore, was patented in 1933. Several methods for the magnetic separation of ground strongly magnetic ores, with the objective of eliminating the forces of molecular and electrostatic cohesion existing between magnetic and nonmagnetic particles by increasing the speed of drum rotation, by the creation of high-frequency magnetic fields and their superposition on steady magnetic field, were also proposed. 
Reduction of the cohesion forces between magnetic and non-magnetic particles can be achieved by increasing the centrifugal force. Re-orientation of the magnetic chains takes place when frequent alternation of the polarity of the magnetic poles is introduced, while the magnetic force holds magnetic particles on the surface of the rotating drum.

Magnetic assemblies of permanent magnets, with a small pole pitch, were applied in the separators of Martsell (developed in 1950) and of Laurilla (1953), for the separation of finely ground magnetite ores. Small and coarse flocs consist of a large amount of magnetic particles and a small portion of dia- and paramagnetic particles. When high-speed rotation of a non-magnetic drum is applied, the flocs break into fragments, as a result of their periodic reorientation and rotation. As a result, the entrained non-magnetic particles are released. This mechanism results in a good level of separation of the magnetics from the non-magnetics.

More recently, most iron-ore beneficiation plants employed magnetic separators that were developed during the 1950s [4,5]. Modernization of these machines during the last few years was associated mainly with the appearance of new materials and with the improvement of production technology.

Why are we posing the question: what is the next step?

All these machines have proved to be highly reliable, which is confirmed by their longevity. These machines possess a high throughput and ensure a high recovery of magnetic material. It would thus seem that the best machines could be only those with a higher throughput. However, over the decades of industrial application of magnetic separators from the moment of their principal development, many things have changed. As a result of reductions in the raw material base, energy shortages and environmental problems required a more thorough review of the existing level of the technology employed in ore beneficiation, aimed at their rationalization and improvement [1].

Since a technology is realized by means of the machines that are available, the matter of technological possibilities is inherently associated with technological potentialities of the processing equipment. To assess the level of development of magnetic separators that have been recently applied on industrial scale, it is necessary to analyse their role and position in the processing technology.

\section{EXISTING APPROACHES TO THE UPGRADING OF IRON CONCENTRATES}

Analysis of the processing flowsheets for magnetite (taconite) ores shows that concentrates upgrading is achieved exclusively through special finishing operations such as fine screening, flotation, desliming and combination of these operations, and also by means of their more thorough (fine) comminution and subsequent magnetic separation combined with desliming [6,7].

As an example, the finishing flowsheets applied at a number of concentrating plants in Russia are considered. Magnetic finishing flowsheets with fine grinding are applied at the Lebedinskiy, Mikhaylovskiy and Olenegorskiy Mine Combines.

At the Mikhaylovskiy Mine Combine, the iron content is upgraded from $66 \%$ to $66.7 \%$, after regrinding and supplementary magnetic separation, as is shown in Fig. 1 [7]. At the Olenegorskiy Mine Combine (OLKON), in order to increase the 


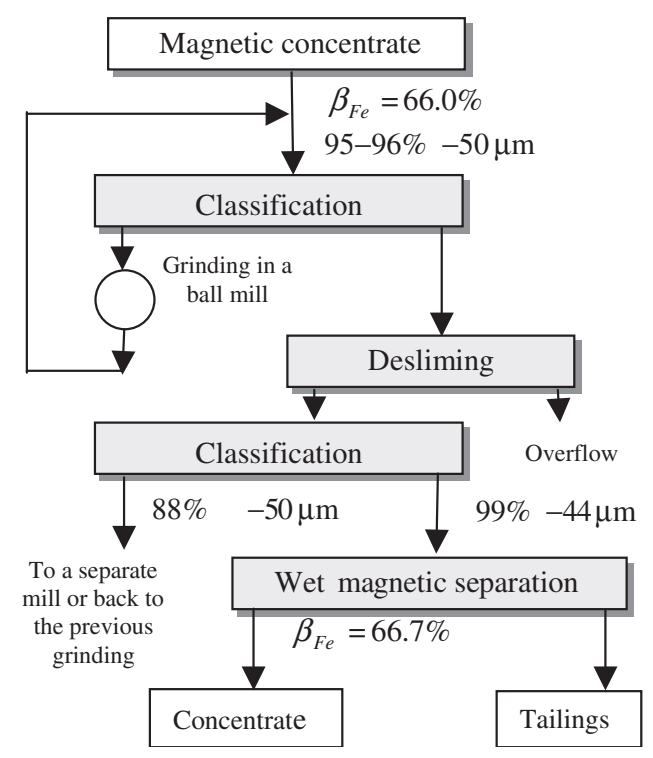

FIGURE 1 Processing flowsheet of perfecting the concentrates at Mikhailovsky Mining Combine.

iron content from $63.6-65 \%$ up to $66.7-66.6 \%$, improvement of rough concentrate is organized at an individual operational circuit (line).

The rough concentrates, from the first to the sixth, and from the eighth to the 12 th line (separately) are reground at the seventh operation line and then processed by magnetic separation, as shown in Fig. 2.

The production of high-grade concentrates, with an iron content of $70 \%$, from the magnetite concentrates, destined for metallic pellets production, is introduced at the finishing circuit of the Lebedinskiy Mine Combine. The flowsheet includes regrinding, magnetic desliming and several steps of magnetic separation, as shown in Fig. 3.

Production of superconcentrate at the Olenegorskiy Mine Combine includes regrinding of rough concentrate, magnetic separation and magnetic hydro-dynamic separation (MHDS), as is illustrated in Fig. 4.

It is noteworthy that the use of very fine grinding for rougher concentrates increases energy consumption. Furthermore, moisture in the filtration cake of the final concentrate increases, which results in an increase of flux consumption during pelletizing and a decrease of iron content in the pellets.

It is not out of place to consider energy consumption in a greater detail. As can be seen from Fig. 5, specific energy consumption in the grinding operations is the highest of all general ore dressing stages and amounts to approximately two thirds of the total energy consumption. Magnetic separation energy consumption is one of the lowest of all stages and does not exceed $2 \%$ [2,8-10].

Analysis of the processing flowsheets for magnetite (taconite) ores production and investigation of the limitations of the process show that the energy consumption in grinding can be reduced by $10-20 \%$, as a result of changing the fundamental approach to and reassessment of the role of magnetic separation. 


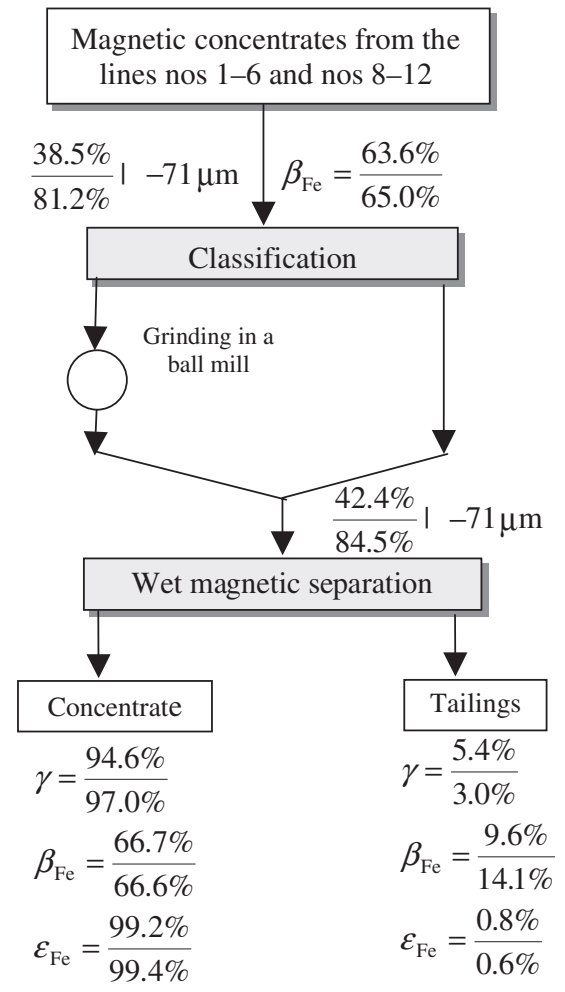

FIGURE 2 Processing flowsheet of the concentrates perfecting line (the 7th line) of concentrating plant at 'OLKON'.

\section{ANALYSIS OF THE PROCESSING TECHNOLOGY FOR MAGNETITE ORES: LIMITATIONS OF THE TECHNOLOGY}

Results of the application of similar process flowsheets for the final concentrate finishing appear to be excellent. They are, indeed, quite good, taking into account the level of the existing technology. Results are acceptable, if we do not take into account the fact that the material coming into the finishing circuits, to undergo further regrinding, already consists of $92-95 \%$ liberated magnetite, and only of $5-8 \%$ of middlings and inter-grown particles of magnetite with gangue. It is only the latter material which, strictly speaking, requires recovery by regrinding $[4,6,11,12]$.

The functional performance of technological operations and their efficiency can be seen sufficiently clearly when considering and analysing a phase composition of the products from the process operations. Such an analysis allows determination of the efficacy of any processing procedure operates.

Analysis of the technology and the phase composition of products from the process operations reveals imperfection of the classifying function of hydrocyclones as the cause of a large number of shortcomings in the current processing technology $[1,2,11,13,14]$. The efficiency of classification of ground products by the degree of grain liberation is one of the general conditions for obtaining high-grade concentrates.

Spiral classifiers and hydrocyclones have been widely used at various operating plants. Both types of classifying devices offer insufficient separation effectiveness. 


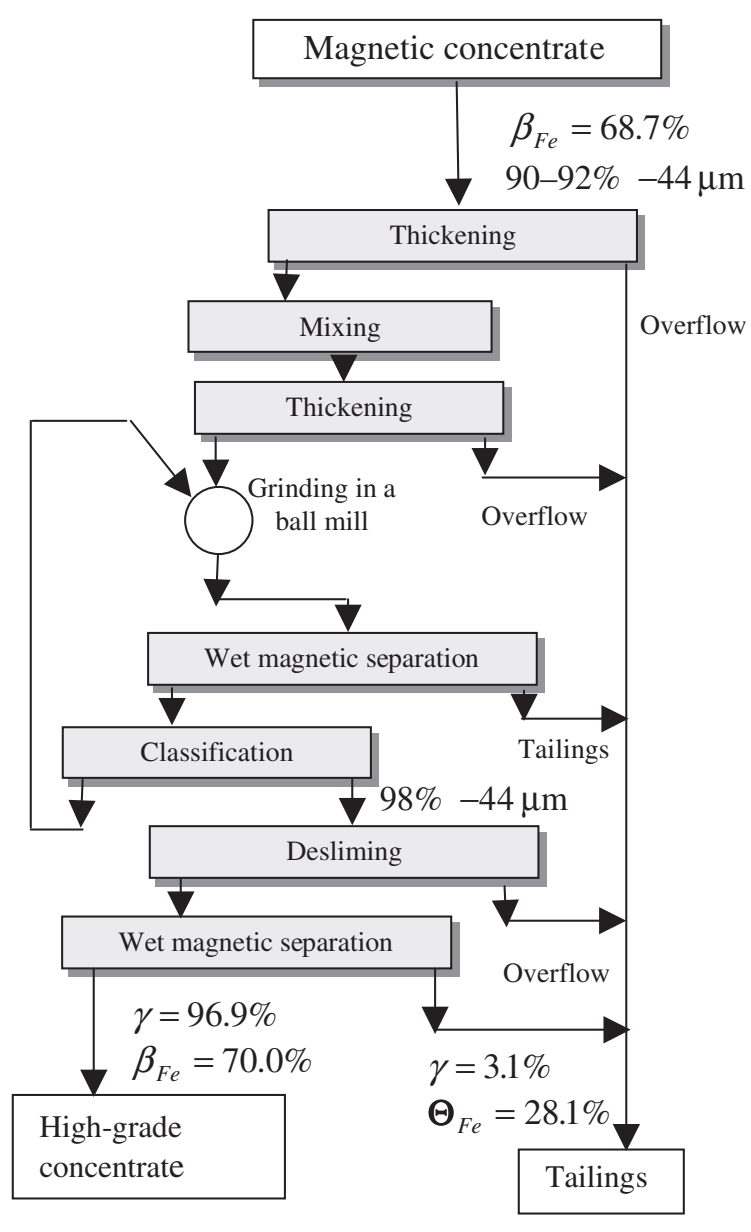

FIGURE 3 Processing flowsheet of perfecting the concentrates at Lebedinskiy Mining Combine.

During the classification process, the minerals are divided according to size and density, which results in obstruction of the product (overflow) with coarse (but poor in iron content) inter-grown particles. Furthermore, a significant percentage of already liberated grains is circulated within the grinding circles.

A summary of the products of hydrocyclone segregation by size at the second classification stage of the Kostomuksha Mine Combine is given in Tables I and II. A fraction composition of the separation product from hydrocyclones at the Kostomuksha Mine Combine is shown in Fig. 6. The underflow is returned for regrinding in the mill. The following are clear from Fig. 6:

(1) The degree to which the hydrocyclone overflow consists of particles of the control size and the degree to which it is contaminated by inter-grown particles.

(2) The degree to which the hydrocyclone underflow consists of coarse intergrown particles and the degree to which it is 'contaminated' with particles of the control size. 


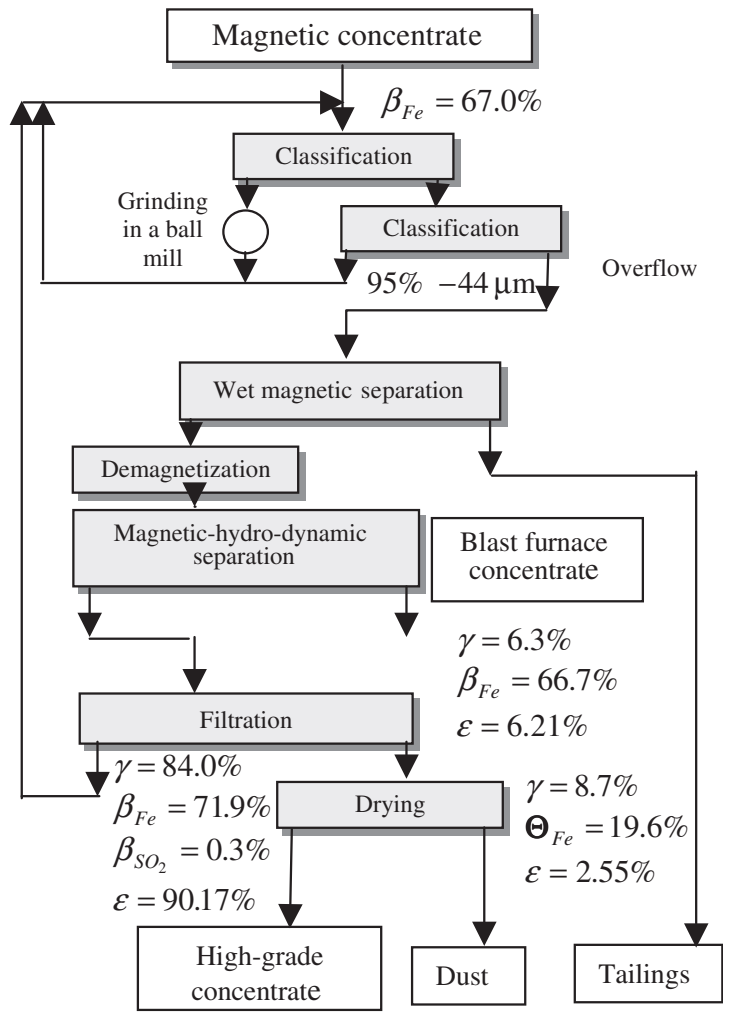

FIGURE 4 Processing flowsheet for obtaining a super-concentrate at 'OLKON'.

Therefore, when speaking about the hydrocyclone underflow, it is important to note the following:

(1) Fraction $-0.2+0.1 \mathrm{~mm}$ consists of inter-grown particles with $20-25 \% \mathrm{Fe}$ (the yield into the fraction is only $7.1 \%$ );

(2) Fraction $-0.05 \mathrm{~mm}$ consists of the already liberated particles of magnetite and averages $69-72 \% \mathrm{Fe}$ (the yield into the fraction is $64 \%$ );

(3) Fraction $-0.1+0.05 \mathrm{~mm}$ averages $63-65 \% \mathrm{Fe}$ and consists, to a large extent, of coarse liberated particles of magnetite (the yield into the fraction is $28.3 \%$ );

(4) The yield into the fraction of $-0.4+0.2 \mathrm{~mm}$ is very small (in our instance, it is $0.6 \%$ ); it consists of the medium and rich inter-grown particles and averages $55-57 \% \mathrm{Fe}$.

Conversely, the hydrocyclone overflow consists of inter-grown particles of fraction $-0.2+0.05 \mathrm{~mm}$ (the yield into the fraction is $9 \%$ ), the Fe content of which ranges from 17 to $25 \%$.

Relatively coarse and low-grade particles in the classification overflow pose several difficulties for subsequent technological processes downstream. Being sufficiently heavy, owing to their coarse size, they report into the underflow (sands) during the subsequent desliming. However, when repeated attempts to classify them by a hydrocyclone are made, the probability of their recovery into the hydrocyclone 


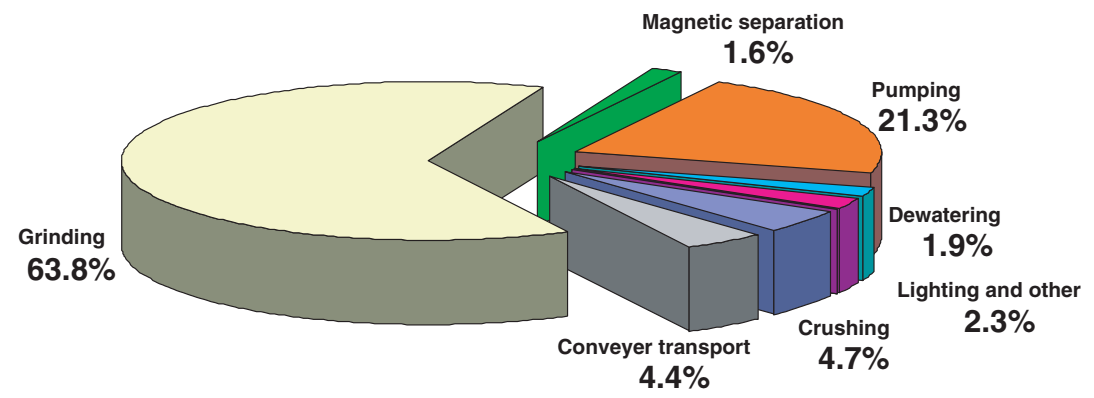

FIGURE 5 Distribution of energy consumption in an ore-dressing process.

\section{Fraction compound of the} hydrocyclones overflow

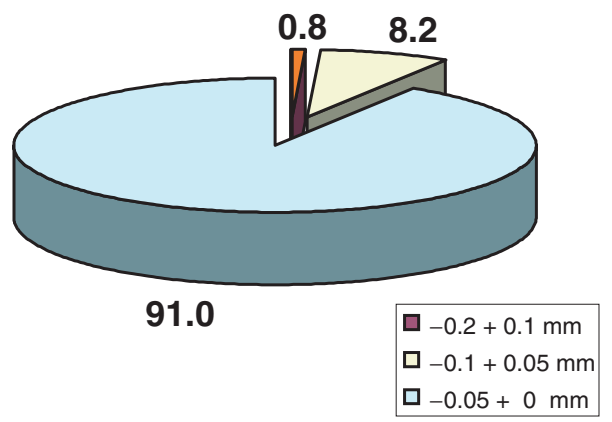

Fraction compound of the hydrocyclones underflow

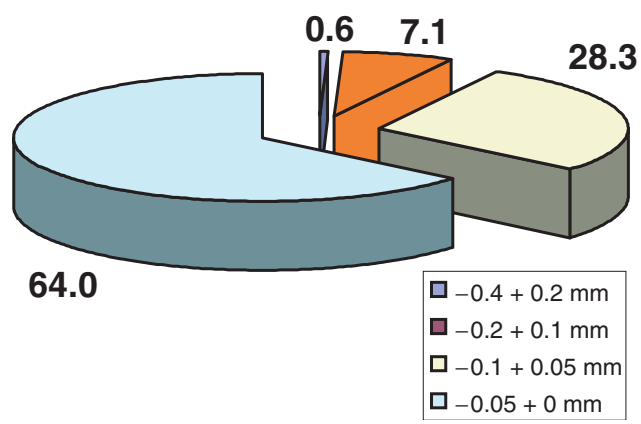

FIGURE 6 Fraction compound of the products of classification by a $500 \mathrm{~mm}$ hydrocyclone.

overflow is again very strong. These inter-grown particles have a sufficiently high iron content not to be rejected into the tailings and they inevitably report into the magnetic product.

It is clear from an example of one of the best enterprises in this field that in the existing technological flowsheets, while attempting to obtain fine hydrocyclone 
TABLE I Summary of fractions in the hydrocyclone overflow

\begin{tabular}{lcc}
\hline Size fractions & Yield $(\%)$ & Fe content $(\%)$ \\
\hline$-0.2+0.1 \mathrm{~mm}$ & 0.8 & 24.34 \\
$-0.1+0.05 \mathrm{~mm}$ & 8.2 & 23.24 \\
$-0.005 \mathrm{~mm}$ & 91.0 & 66.43 \\
Total & 100.0 & 62.55 \\
\hline
\end{tabular}

TABLE II Summary of fractions in the hydrocyclone underflow

\begin{tabular}{lcc}
\hline Size fractions & Yield $(\%)$ & Fe content $(\%)$ \\
\hline$-0.4+0.2 \mathrm{~mm}$ & 0.6 & 57.26 \\
$-0.2+0.1 \mathrm{~mm}$ & 7.1 & 23.45 \\
$-0.1+0.05 \mathrm{~mm}$ & 28.3 & 63.58 \\
$-0.005 \mathrm{~mm}$ & 64.0 & 71.06 \\
Total & 100.0 & 65.48 \\
\hline
\end{tabular}

overflow, a large quantity of liberated magnetite in the hydrocyclone underflow is returned into the regrinding stage.

It is evident that fine screening is not a panacea either. As can be seen from the grain size distribution in the hydrocyclone overflow, particles in the $-0.1+0.05 \mathrm{~mm}$ fraction, with $23 \% \mathrm{Fe}$, reporting into the undersize, make it unavoidably necessary to add one more supplementary treatment operation for the material contaminated in a such manner. Reduction of the cut size by further classification down to $0.05 \mathrm{~mm}$ will result in redirecting coarse magnetite grains in the $-0.1+0.05 \mathrm{~mm}$ fraction (the amount of which, judging by the grain size distribution of the underflow, is rather high) back to the mill. This last circumstance limits possible gains.

Analysis of the composition of the products of the technological operations shows that considerable reserves are associated with the existing process operations. A technical solution to these problems, changes to technological approaches, will allow, in principle, one to come close to the basic beneficiation principle 'do not over-crush, do not over-grind' that, following from the above analysis, is not adhered to in the existing process technology.

An alternative for the existing process technology is to apply apparatus that allows for the division of material according to magnetic properties, rather than the widely used hydrocyclones and size classification. However, under its existing conditions, the process of magnetic separation is also far from fully satisfactory.

\section{THE MAIN REQUIREMENT FOR A NEW-GENERATION SEPARATOR IS INCREASED SELECTIVITY}

The presently applied wet drum magnetic separators for treatment of magnetite ores, posses low selectivity of segregation at all processing stages $[4,15]$.

In this sense a comparative analysis of the features of the mineral phases of the feed and of the magnetic product of wet magnetic separation 2 at the concentrator of the Kostomuksha Mine Combine is very demonstrative. 
The data on contents of mineral phases of these products are given below:

- liberated magnetite: grains of $100 \%$ magnetite;

- rich magnetite inter-grown particles: consist of $90 \%$ magnetite and $10 \%$ quartz;

- middle magnetite inter-grown particles: consist of $50 \%$ magnetite and $50 \%$ quartz;

- poor magnetite inter-grown particles: consist of 10\% magnetite and $90 \%$ quartz;

- liberated quartz: grains of $100 \%$ quartz.

These mineral phase features in the feed and in the magnetic product of the wet magnetic separation 2 are presented in Tables III and IV, and in Figs. 7 and 8.

Analysis of results shows that after passing through the magnetic separation the ore material mostly gets rid of the poor inter-grown particles and liberated quartz as well. The portion of poor inter-grown grains in the processing material was reduced from 31.5 to $15.5 \%$. The last circumstance shows that the ability of the existing magnetic separators to clean a magnetic product, even from recovered quartz, is limited. The portion of middle inter-grown grains was reduced from $3.5 \%$ to $2 \%$. The increase of the portion of the recovered magnetite grains from $54 \%$ to $73 \%$ is attributed to the rejection of practically $50 \%$ of poor inter-grown grains. The change in the concentration of rich inter-grown grains is negligible (from $6 \%$ to $6.5 \%$ ).

Transformation of composition of the mineral phase of the ore material by passing it through magnetic separation 2 is clearly presented in Fig. 9. It can be seen that while standard magnetic separators have a relatively high specific throughput, they cannot ensure high technological efficiency.

Reduction of the magnetic concentrate grade, as a result of entrainment of nonmagnetic grains in magnetic flocs, places additional demand on the design of the separator, in order to ensure floc breakage and the release of non-magnetic and inter-grown grains [4].

Elimination of magnetic product contamination with liberated gangue, and nonliberated ore-gangue inter-grown particles still remains the most important problem

TABLE III Mass concentration of mineral phases in the feed into wet magnetic separation

\begin{tabular}{|c|c|c|c|}
\hline \multirow[t]{2}{*}{ Liberated } & \multicolumn{2}{|c|}{ Magnetite } & \multirow{2}{*}{$\frac{\text { Quartz }}{\text { Poor intergrown particles }}$} \\
\hline & Rich intergrown particles & Middle intergrown particles & \\
\hline $100 \%$ magnetite & $\begin{array}{l}90 \% \text { magnetite } \\
10 \% \text { quartz }\end{array}$ & $\begin{array}{l}50 \% \text { magnetite } \\
50 \% \text { quartz }\end{array}$ & $\begin{array}{l}10 \% \text { magnetite } \\
90 \% \text { quartz }\end{array}$ \\
\hline $54 \%$ & $6 \%$ & $3.5 \%$ & $31.5 \%$ \\
\hline
\end{tabular}

TABLE IV Mass concentration of mineral phases in magnetic product from wet magnetic

\begin{tabular}{|c|c|c|c|}
\hline \multirow[t]{2}{*}{ Liberated } & \multicolumn{2}{|c|}{ Magnetite } & \multirow{2}{*}{$\frac{\text { Quartz }}{\text { Poor intergrown particles }}$} \\
\hline & Rich intergrown particles & Middle intergrown particles & \\
\hline $100 \%$ magnetite & $\begin{array}{l}90 \% \text { magnetite } \\
10 \% \text { quartz }\end{array}$ & $\begin{array}{l}50 \% \text { magnetite } \\
50 \% \text { quartz }\end{array}$ & $\begin{array}{l}10 \% \text { magnetite } \\
90 \% \text { quartz }\end{array}$ \\
\hline $73 \%$ & $6.5 \%$ & $2 \%$ & $15.5 \%$ \\
\hline
\end{tabular}




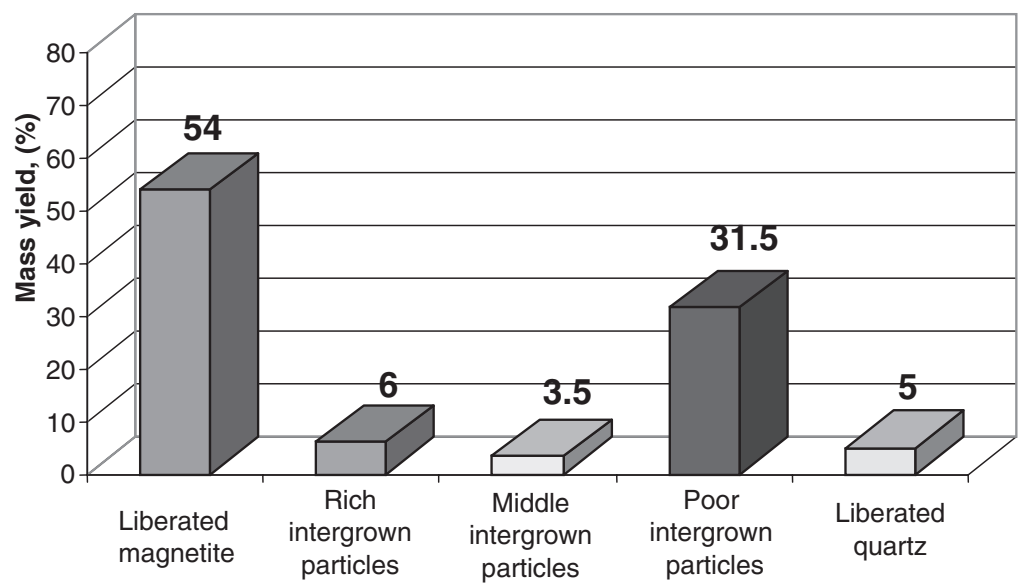

FIGURE 7 Characteristics of the mineral phases in the feed into a wet magnetic separation 2.

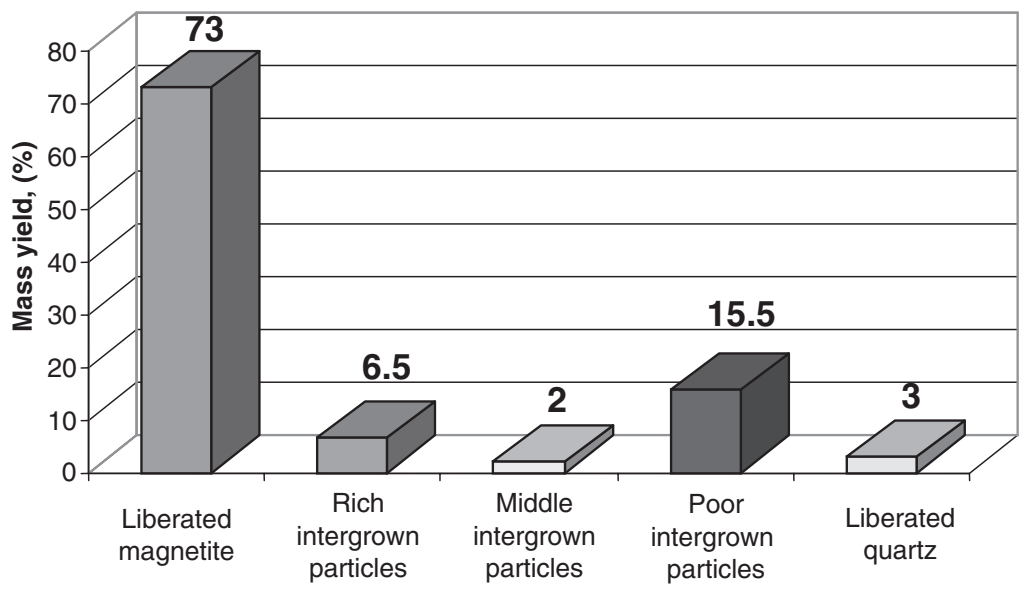

FIGURE 8 Characteristics of mineral phases in the magnetic product from a wet magnetic separation 2.

to be solved. Realization of this reserve would allow us to upgrade the magnetite concentrates and to improve the economy of the entire iron ore beneficiation process.

Introduction of highly selective drum magnetic separators, in order to achieve recovery of fine disseminated inter-grown particles and already liberated magnetic particles into separate products, is therefore of significant current interest.

This conclusion leads to a conception that there is considerable need for revision of the concept of magnetic beneficiation and also the traditional approach to the design of magnetic separators, which is based on maximization of the recovery of the mineral value into the magnetic product.

Already in the middle of 1970 s a conclusion was being made concerning the necessity of a greater attention to the improvement of actual technological flowsheets. In order to realize such an improved process, it is, therefore, necessary to accelerate development efforts that would result in the creation and introduction of new models of magnetic separators. 


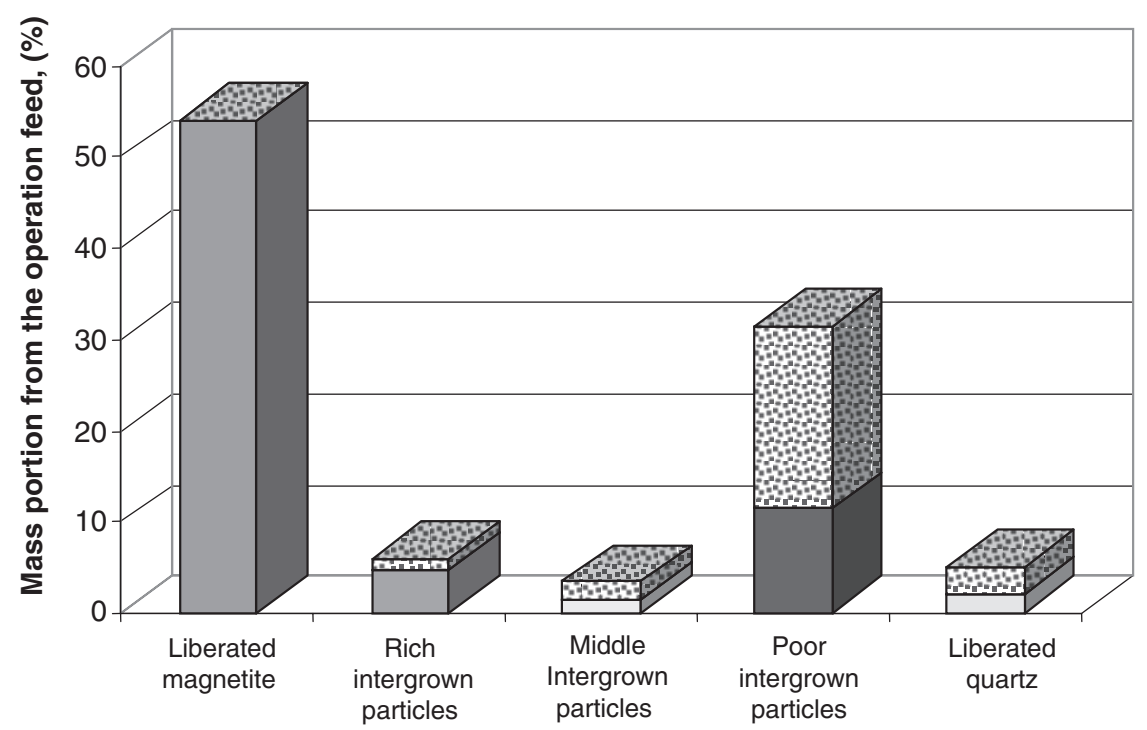

FIGURE 9 Absolute alteration of the mineral-phase compound of the ore material to be treated by magnetic separation 2. The dotted sections denote material rejected into the process tailings.

Thorough investigation of the existing process flowsheets for the treatment of magnetite ores and also detailed analysis of their disadvantages allow us to assume that stable stereotypes, which were established in 1960s, will be surpassed in the near future.

The process flowsheets of the existing magnetite ores concentrating plants are fairly typical. The main approach to the processing of taconite was developed in the 1940s and 1950s, when the basic taconite processing flowsheet was invented, and this is still used today [5].

Technical inefficiencies and limitations are also typical. During the last few decades disadvantages of the applied process flowsheets have become habitual. This is why the usual approach to the design of new equipment for magnetic separation has suffered from stereotypes formed previously.

One of the general principal requirements for the design of magnetic separators is to maximize the recovery of the magnetic component into the magnetic product. The maximization of the recovery, together with the existing parallel trend to develop equipment with a higher throughput, does not allow for any other method.

The influence of these stereotypes has found its reflection in the models of many present-day developers. Creation of such models was accelerated by the appearance, in recent years, of powerful magnetic materials with high magnetic energy. Numerous changes, both in the conditions under which the process is conducted (such as lowering the grade of the ore and increasing the energy prices) and nowadays growing requirements for the product bring forward new priorities based on a complex approach and understanding the necessity to solve technological tasks in linkage together with the creation of new equipment, capable of resolving these new specific tasks. Such an approach may require an entire revision of traditional principles.

An ideal future magnetic separator would, in the first place, be a highly selective machine capable of separating liberated magnetite grains from rich inter-grown particles of magnetite with gangue. This problem has not been solved successfully, 
as yet, particularly in wet processing. The importance of solving this problem can be explained by the fact that in the overwhelming majority of iron ore beneficiation plants, treatment is accomplished by the wet method. It is noteworthy that the new generation separators will not completely displace the existing ones. Standard separators will be used, as usual, to reject gangue into the tailings, while the magnetic product will be re-treated by selective cleaning separators. This primary magnetic concentrate will then be separated into the middlings fraction and the high-grade concentrate, with a quality close to that of the final concentrate.

Selection of the magnetic material by degree of liberation will allow for the process optimization to be achieved in almost all important parameters. It will allow us to save the energy consumed in the grinding process, to increase the grain size in the final concentrate, to avoid the over-grinding and to improve the conditions for filtration and palletizing.

It has thus been established that the necessity to develop a magnetic separator with novel qualitative capabilities is inevitable. Such a separator will be accepted by the industry in a near future, as soon as the understanding of importance of its advantages becomes clear to the production mindset.

\section{THE INITIAL RESULTS OF THE NOVEL SELECTIVE SEPARATOR}

The authors of this paper have been investigating such energy-saving problems for over a decade. During this period, they have developed separators for selective separation of the middling product. The main principles of the energy-saving approach to the beneficiation of magnetite quartzite (taconite) ores have been discussed in a number of publications [2,12]. However, in the absence of a market-based economy in Russia at that time, these ideas had not been implemented. For instance, the price of one kilowatt-hour of electricity was disproportionally set at the level below US\$0.03. Such an unjustified low price did not stimulate any energy saving. Therefore, since we started developing new concepts of magnetic separators long before the term 'energy saving' became fashionable, a separator of new generation has recently become available.

A prototype of the novel selective separator (superconcentrator) has been tested in the processing facilities of the concentrating plant at the Kostomuksha Mine Combine. The test separator achieves magnetic flocs breakage by applying an alternating magnetic field of increased frequency, in contrast to standard models.

The separator tests have shown promising results. Magnetic product with an iron content of $68 \%$ has been extracted from the feed into the tertiary grinding mill assaying $62 \% \mathrm{Fe}$. Therefore, the possibility of removing a portion of a well-conditioned material from the grinding process has been proved. A partial relief of the operating capacity of the grinding equipment ensures significantly better conditions for the grinding, namely: it eliminates over-grinding of liberated magnetite grains, increases the size of the conditioned product and, at the same time, improves the conditions for liberation of the inter-grown particles.

\section{CONCLUSION}

Analysis of product composition of the process procedures shows that the existing technology of beneficiation of magnetite (taconite) ores has numerous reserves for 
improvement. Technical solution of these problems, the change of the technological approach will allow us, in principle, to get closer to the basic beneficiation principle 'do not over-crush, do not over-grind' which, following from the above analysis, is not adhered to in the existing process technology.

As an alternative to the existing process technology, separation of material according to magnetic properties can be employed, instead of widely using hydrocyclones and size classification.

The magnetic separator of tomorrow is, in the first place, a highly selective separator possessing the ability to separate liberated (uncovered) magnetite grains from rich inter-grown particles of magnetite with gangue. This is the only way of unravelling and utilizing the reserves of the imperfect existing process flowsheets. This new approach ensures relief from the grinding capacities, since both gangue and liberated value mineral no longer require further regrinding.

A partial relief of the operating capacity of the grinding equipment ensures considerably better conditions for grinding. With the introduction of these highly selective separators, the existing concentration flowsheets, which, as we have seen, use expensive, wasteful technological approach, will become obsolete.

New promising technological flowsheets will be based on the stage-by-stage extraction of the material of a high grade, on the optimization of the mill feed according to phase composition (reduction of the percentage of liberated magnetite, and increasing the percentage of the middlings).

Selection of magnetic material according to liberation grade will allow for process optimization in all most important parameters: it will save the energy consumed during the grinding process; it will reduce the losses, into the operations tailings, of over-ground magnetite; it will increase the grain size in the final concentrate; and by avoiding over-grinding, it will improve the conditions for the filtration and pelletization.

Therefore, the necessity of creating the magnetic separator with brand-new qualitative potentialities has become imminent, and the separator will be claimed by the industry in the near future.

The newly designed selective magnetic separator has allowed us to extract, in a wet mode, a high-grade magnetic product from the feed into mill. The increased level of selectivity is achieved as a result of modification of the traditional approach and, by overcoming a number of technical problems, complexity of which deemed to be insurmountable in an effort to improve the efficiency of separation.

\section{References}

[1] I. Iwasaki, (1994), Mineral processing to material processing, Min. Eng., 12 1361-1366.

[2] V.V. Karmazin, M.A. Bikbov and A.A. Bikbov, (2002), The energy saving technology of beneficiation of iron ore, Mag. Electr. Sep., 11, 211-224.

[3] V.G. Derkach and I.S. Datsuk, Electromagnetic Processes of Beneficiation, Metallurgizdat, Moscow, 1947.

[4] V.I. Karmazin and V.V. Karmazin, Magnetic Methods of Beneficiation, Nedra, Moscow, 1984.

[5] W.R. Yernberg, (2000), Operational improvements keep Minnesota iron competitive, Min. Eng., 52, 62-63.

[6] B.M. Maliy, B.V. Fidelman, N.N. Bereznoy and E.A. Malaya, (1986), The rational technology of producing of high-grade magnetite concentrates, Gor. J. (Moscow), 9, 42-43.

[7] V.A. Sentemova, (1997), The problem of improving the grade of the concentrates of the iron ore beneficiation plants, Gor. J. (Moscow), 2, 41-45. 
[8] V.I. Revnivtcev, E.A. Hopunov, E.I. Azbel, L.P. Zarogatsky, Selective Disintegration of Minerals, Nedra, Moscow, 1988.

[9] N.I. Sokur, Savings of energy resources in disintegration of mineral raw materials, In V.A. Arsentjev (Ed.): Ways of Resource-Saving in Ferrous Metals Ores Processing, Nedra, Moscow, 1990, p. 3-8.

[10] R.E. McIvor, T.P. Weldum, B.J. Mahoski and R.S. Rasmussen, (2000), Systems approach to grinding improvements at the Tilden concentrator, Min. Eng., 52, 41-47.

[11] P.E. Ostapenko, The Theory and Practice of Magnetite Ores Dressing, Nedra, Moscow, 1985.

[12] A.A. Bikbov, Technological principles of resources-saving on magnetite ore dressing, In V.A. Arsentjev (Ed.): Ways of Resource Savings on Ferrous Metals Ores Processing, Nedra, Moscow, 1990, pp. 24-27.

[13] Z.I. Nekrasov, V.A. Binkevich, V.V. Binkevich and T.N. Kucher, Ways of upgrading iron ore, In G.V. Gubin (Ed.): Beneficiation of Ferrous Metals Ores, Nedra, Moscow, 1975, p. 13-19.

[14] M.A. Bikbov, (1997), Problem of lowering of alkaline metals content in iron ore concentrate, Gor. J. (Moscow), 9, 42-43.

[15] L.G. Bykov, G.S. Chigrin and I.E. Levin, Some questions of improving the selectivity of separation of fine ground magnetite ores, In G.V. Gubin (Ed.): Intensification of the preparation magnetic and gravity processes of beneficiation: theses of reports on conference in Dnepropetrovsk, Nedra, Moscow, 1980.

[16] P.E. Ostapenko, T.T. Berdysheva and N.S. Revzina, Improvement in Quality of Magnetic Concentrates Abroad, Chermetinformatia, Moscow, 1983. 